\title{
Philosophiques
}

\section{Les états intentionnels des créatures solitaires}

\section{Daniel Laurier}

Volume 14, numéro 2, automne 1987

URI : https://id.erudit.org/iderudit/027016ar

DOI : https://doi.org/10.7202/027016ar

Aller au sommaire du numéro

\section{Éditeur(s)}

Société de philosophie du Québec

\section{ISSN}

0316-2923 (imprimé)

1492-1391 (numérique)

Découvrir la revue

\section{Citer cet article}

Laurier, D. (1987). Les états intentionnels des créatures solitaires. Philosophiques, 14(2), 229-359. https://doi.org/10.7202/027016ar

\section{Résumé de l'article}

Je soutiens qu'il y a deux façons d'individuer les états intentionnels de créatures qui sont dépourvues de toute compétence linguistique, à savoir par leur rôle propositionnel ou par leurs conditions de vérité, mais que cette distinction ne vaut que pour les états intentionnels singuliers. L'examine ensuite différentes façons de spécifier, tout en restant dans le cadre d'une conception représentationnaliste de l'intentionnalité, les conditions de vérité des attributions d'états intentionnels privés (ou sublinguistiques) du langage ordinaire selon le mode d'individuation considéré. Il s'avère qu'une attribution d'état intentionnel singulier ne peut identifier simultanément le rôle propositionnel et les conditions de vérité des états intentionnels attribués, et que la nécessité d'invoquer des états intentionnels de se pour expliquer les comportements menace de remettre en question la thèse représentationnaliste. 


\title{
LES ÉTATS INTENTIONNELS DES CRÉATURES SOLITAIRES
}

\author{
par Daniel Laurier
}

RÉSUMÉ. Je soutiens qu'il y a deux façons d'individuer les états intentionnels de créatures qui sont dépourvues de toute compétence linguistique, à savoir par leur rôle propositionnel ou par leurs conditions de vérité, mais que cette distinction ne vaut que pour les états intentionnels singuliers. J'examine ensuite différentes façons de spécifier, tout en restant dans le cadre d'une conception représentationnaliste de l'intentionnalité, les conditions de vérité des attributions d'états intentionnels privés (ou sublinguistiques) du langage ordinaire selon le mode d'individuation considéré. Il s'avère qu'une attribution d'état intentionnel singulier ne peut identifier simultanément le rôle propositionnel et les conditions de vérité des états intentionnels attribués, et que la nécessité d'invoquer des états intentionnels de se pour expliquer les comportements menace de remettre en question la thèse représentationnaliste.

ABSTRACT. I claim that there are two ways of individuating the intentional states of creatures without any kind of linguistic competence, namely, either by their propositional role or by their truth-conditions, but that this distinction holds only for singular intentional states. I then consider several ways of specifying, within the limits of a representational theory of intentionality, the truth-conditions of statements attributing private (or sublinguistic) intentional states according to the mode of individuation chosen. It turns out that such statements cannot simultaneously identify both the propositional role and the truth-conditions of the intentional states attributed, and that the need to invoke de se intentional states in order to explain behavior seems to put the representational theory into difficulty.

«Never mind mind, essence is not essential and matter doesn't matter ».

Nelson Goodman 
Les philosophes du langage ont l'habitude d'aborder le problème du sens des attributions d'états intentionnels sous l'angle de sa compositionnalité ou de sa dérivabilité dans tel ou tel cadre théorique déterminé à l'avance. Étant données certaines hypothèses générales sur la forme que doit prendre une théorie du sens adéquate et certaines intuitions sur le ou les sens qu'il convient d'assigner aux énoncés d'attitudes propositionnelles, il s'agit alors de trouver les règles de composition ou de dérivation et les catégories et valeurs sémantiques des expressions primitives qui permettront de rendre compte des intuitions de départ sans s'écarter du cadre formel privilégié. Une telle entreprise peut prendre deux formes principales (et complémentaires) selon qu'elle s'intéresse au sens conventionnel ou au sens intentionnel des attributions d'états intentionnels. Dans le premier cas l'intérêt portera sur la compositionnalité du sens conventionnel de ces énoncés, c'est-à-dire sur la façon dont il dépend de celui de leurs constituants et de paramètres contextuels bien définis. Il y a évidemment place ici pour différentes conceptions de la forme que doit prendre une théorie du sens conventionnel ; les principales conceptions rivales étant celles qui privilégient la théorie tarskienne de la vérité, la théorie des modèles (avec ou sans mondes possibles), et la théorie des rôles conceptuels (ou des conditions d'assertabilité), respectivement. Dans le second cas, le problème sera surtout d'expliciter les maximes pragmatiques et les présuppositions contextuelles qui permettront d'inférer le (ou les) sens intentionnel(s) voulu(s), à partir du sens conventionnel déterminé par une théorie du sens conventionnel supposée donnée.

À mon avis l'échec apparent de toutes les tentatives s'inscrivant dans la perspecrive générale que je viens d'indiquer s'explique moins par l'insuffisance des ressources formelles mises en cuvre que par la précarité ou la confusion (ou l'indétermination) des intuitions sémantiques sur lesquelles elles prétendent s'appuyer. Quoi qu'il en soit, les pages qui suivent visent essentiellement à préciser, si possible, ces intuitions, en s'interrogeant sur les conditions de vérité des attributions d'états intentionnels du langage ordinaire, c'est-à-dire sur la nature des états intentionnels eux-mêmes, sans se soucier des mécanismes formels par lesquels on pourrait éventuellement leur assigner de façon systématique leurs conditions de vérité. J'ai bon espoir qu'une telle enquête sera riche d'enseignements sur la façon dont il convient d'aborder la question de l'interprétation radicale et celle du statut de la psychologie populaire. 
Ma thèse principale est qu'il y a deux catégories d'états intentionnels, les états intentionnels privés et les états intentionnels publics, et que les états intentionnels de chacune de ces catégories ont un double aspect, interne et externe. J'espère éventuellement pouvoir montrer qu'il est légitime de s'appuyer sur une théorie des états intentionnels privés pour rendre compte des états intentionnels publics, et que les concepts de la psychologie populaire ne sont ni éliminables ni fondés rationnellement. Mais il n'est évidemment pas possible de développer ici ces idées et je n'ai que des préliminaires à offrir.

En fait il ne sera question dans les pages qui suivent que de la distinction entre les deux aspects des états intentionnels privés, et des conséquences qu'elle peut avoir pour l'interprétation des attributions d'états intentionnels privés. Les autres distinctions font l'objet d'un article publié simultanément dans Recherches sur la philosophie et le langage.

Le présent article est organisé de la façon suivante. Dans la première partie un cadre général de discussion est établi, qui permettra de se demander, dans la deuxième, comment individuer les états intentionnels privés, et dans la troisième, comment interpréter les attributions d'états intentionnels privés. Il convient de garder présent à l'esprit que je compte comme état intentionnel privé un état intentionnel qui peut être attribué à une créature sans présupposer qu'elle ait la capacité de communiquer symboliquement avec ses congénères, ni a fortiori qu'elle dispose d'une langue publique comparable aux langues humaines (ou plus généralement, d'un système symbolique conventionnel). Cela inclut donc les états intentionnels des animaux, et certains des états intentionnels des sujets linguistiques. Je précise aussi que je ne m'intéresserai pas à la légitimité des attributions d'états intentionnels privés, mais seulement à leurs présuppositions, et que je n'entends poser aucune limite, inférieure ou supérieure, au degré d'intelligence dont devrait faire preuve une créature sublinguistique pour qu'on puisse lui attribuer des états intentionnels.

\section{Notions de base}

Il est relativement non problématique de remarquer qu'un locuteur qui énonce une phrase comme 
(1) Fido croit que le chat s'est réfugié dans l'arbre entend normalement i) attribuer à Fido un état intentionnel d'un certain type (en l'occurrence une ctoyance) et ii) identifier cet état à l'aide d'une phrase de son propre langage. En l'occurrence, c'est l'emploi en position complétive de la phrase

(2) Le chat s'est réfugié dans l'arbre qui sert à préciser quelle est la croyance attribuée à Fido. Se demander à quelles conditions la phrase (1) peut être énoncée véridiquement revient alors à se demander quelle relation il doit y avoir entre Fido et la phrase (2) pour que celle-ci puisse être utilisée pour identifier une croyance de Fido. Selon une doctrine assez traditionnelle mais redevenue populaire depuis une quinzaine d'années, il faut (et il suffit) que Fido ait une représentation qui occupe un rôle fonctionnel d'un certain type (caractéristique de la croyance) et qui signifie que (2), ou plus exactement qui ait le sens dans lequel le locuteur utilise (2) lorsqu'il énonce (1). La popularité relative de ce genre de doctrine tient incontestablement à son imprécision et à son extrême généralité. Mon premier objectif est donc d'en rechercher une interprétation plausible.

La première chose à remarquer c'est qu'il doit être suffisant, pour déterminer si une créature a telle ou telle représentation à un moment donné, de la considérer indépendamment de son interaction avec son environnement. En d'autres termes, avoir une représentation, au sens où je souhaite utiliser ce terme, est une propriété interne (i.e., « solipsiste », « étroite ») d'une créature, c'est-à-dire une propriété qui peut être spécifiée sans faire référence à son environnement. Cela implique apparemment qu'à chaque créature (capable d'avoir des états intentionnels) doit correspondre un système de représentations, auquel on peut penser comme à un système syntaxique (i.e., une langue purement formelle) dont les phrases seraient à l'occasion réalisées (i.e., encodées) physiquement dans la créature. Cela revient simplement à dire que des parties de la créature peuvent éventuellement être considérées comme des inscriptions de phrases de ce système syntaxique, parce qu'elles ont la structure requise ${ }^{1}$. Il est à

1. Je n'entends pourtant pas présumer de la réponse générale à la question ontologique du dualisme matière/esprit. Rien ne s'oppose en principe à ce qu'une créature composée d'une substance immatérielle soit organisée de telle façon qu'on puisse y discerner des inscriptions de représentations. Mais une telle possibilité est suffisamment fantaisiste pour être ignorée. Aux partisans (s'il s'en trouve) de cette thèse, je ferai 
noter que deux inscriptions de la même représentation ne partagent pas nécessairement toutes leurs propriétés physiques (autres que leurs coordonnées spatio-temporelles), c'est-à-dire qu'elles peuvent réaliser la même structure formelle sans être physiquement typeidentiques. La mème représentation pourra, par exemple, être réalisée tantôt chimiquement, tantôt électriquement, ou mécaniquement, etc..., particulièrement si on a affaire à deux créatures qui partagent le même système de représentations (quoique rien n'interdise que la même représentation soit encodée sous différentes formes dans la même créature, même simultanément).

Il ne fait aucun doute que la découverte du système de représentations d'une créature (si un tel système existe), doit tenir compte des relations de celles-ci avec l'environnement, et soulève de ce fait un problème méthodologique très considérable ${ }^{2}$, mais que je souhaite ignorer pour le moment. Je me contenterai provisoirement de supposer qu'un tel système a été identifié pour chaque créature, ou du moins existe.

Le comportement d'une créature à un moment donné est déterminé (pour ce qui nous concerne, car les états qui ne sont pas des représentations peuvent aussi être pertinents) par l'ensemble et l'organisation des inscriptions qui y sont réalisées à ce moment, qui sont à leur tour déterminés par les stimuli qui sont enregistrés à ce moment. Autrement dit chaque inscription d'une créature contribue à déterminer la façon dont celle-ci répond à ses stimuli, et c'est cette contribution qui constitue ce qu'on appelle son rôle fonctionnel (ou causal). Le rôle fonctionnel d'une inscription correspond ainsi, intuitivement, à l'ensemble de ses relations causales avec les stimuli, les réponses, et les autres inscriptions de la créature. Cette brève caractérisation doit maintenant être précisée de diverses manières.

On noterá d'abord que la notion de rôle fonctionnel peut s'entendre de façon solipsiste ou non solipsiste, selon que les stimuli et les réponses sont conçus comme des modifications de la créature (p. ex., comme des excitations des surfaces sensorielles ou

simplement remarquer que mes propos devraient s'appliquer, mutatus mutandis, aux archanges comme aux robots et aux animaux.

2. Je songe ici, en particulier, à la dimension normatıve de l'entreprise, et au statut des présuppositions de rationalité ou de fiabilité qu'elle doit vraisemblablement faire intervenir. 
des extrémités mobiles) ou comme des éléments de l'environnement. C'est du rôle fonctionnel au sens solipsiste qu'il s'agit dans ce qui suit. D'autre part, une inscription est un particulier, qui a été causé par certains stimuli et certaines autres inscriptions, et qui causera des réponses et de nouvelles inscriptions. Cette histoire causale d'une inscription (c'est-à-dire, la liste de ses causes et de ses effets réels) ne doit pas être confondue avec son rôle fonctionnel, qui est une propriété dispositionnelle, et englobe l'ensemble de ses relations causales possibles (c'est-à-dire, essentiellement, l'ensemble des effets qu'elle pourrait avoir dans telles ou telles conditions ; on peut ici ignorer ses causes, puisque dès lors que l'inscription existe, ses causes sont déterminées).

Comme je l'ai remarqué, c'est l'ensemble et l'organisation des inscriptions d'une créature qui déterminent, conjointement, son comportement. C'est dire qu'une inscription donnée pourra contribuer à provoquer une réponse différente au même stimulus en fonction des autres inscriptions réalisées au moment de la stimulation et de ses relations avec elles. Il faut donc vraisemblablement distinguer le rôle fonctionnel « absolu » d'une inscription et son rôle fonctionnel « relatif » (i.e., relatif à une configuration d'inscriptions donnée). Je ne puis mieux faire, pour faire comprendre cette distinction, que de comparer le rôle fonctionnel absolu d'une inscription au rôle inférentiel d'une phrase dans un système de déduction naturelle, c'est-à-dire à l'ensemble des dérivations où cette phrase a une occurrence essentielle qu'on peut construire dans ce système. Le rôle fonctionnel relatif serait pour sa part comparable au rôle inférentiel d'une phrase dans une théorie axiomatique, c'est-à-dire à l'ensemble des dérivations où cette phrase a une occurrence essentielle qu'on peut construire dans le système déductif sousjacent de cette théorie et dont les prémisses incluent tous les axiomes de la théorie. Cette distinction est ainsi analogue à la distinction entre la structure déductive d'une langue et celle des théories formulées dans cette langue.

Cette analogie a cependant l'inconvénient de faire abstraction des relations des inscriptions aux stimuli et aux réponses, qui font aussi partie de leur rôle fonctionnel (qu'on doit ainsi considérer comme une disposition complexe et conditionnelle). Mais surtout elle ne tient pas compte du fait que le rôle fonctionnel d'une inscription ne dépend pas seulement de la représentation dont elle 
est une occurrence, mais aussi du mode sous lequel cette représentation est réalisée. En effet le rôle fonctionnel d'une inscription ne sera pas le même selon qu'elle a la valeur d'une croyance ou celle d'un désir. Un rôle fonctionnel a donc deux aspects ou composantes, à savoir, un aspect «propositionnel» (ou « conceptuel») et un aspect modal; or l'analogie du paragraphe précédent ignore l'aspect modal. Je limite ici l'attention à deux modes, correspondant respectivement à la croyance et au désir, que par manque d'imagination j'appelle le mode $\mathrm{C}$ et le mode $\mathrm{D}$. L'importance de ces deux modes tient évidemment à la place centrale qu'occupent les croyances (ou degrés de croyance) et les désirs (ou degrés de désir) dans la façon dont la psychologie populaire explique les comportements. Intuitivement, une inscription sera en mode $\mathrm{C}$ ou en mode $\mathrm{D}$ selon que son rôle fonctionnel est du type associé aux croyances ou aux désirs dans la théorie traditionnelle de la décision (qu'on peut considérer comme une extension et une formalisation d'une partie de ce corps de généralisations qui constituent la psychologie populaire). Il va sans dire que les modes, comme les représentations, doivent être réalisées physiquement dans les créatures.

Il est intéressant de noter qu'une représentation se confond à toutes fins pratiques avec le rôle propositionnel (absolu) de ses inscriptions, c'est-à-dire avec sa place dans le système de représentations de la créature donnée. On aurait donc tort de suivre Dennett (1982) lorsqu'il suggère de considérer les attitudes propositionnelles comme des relations à des contenus «notionnels» plutôt que comme des relations à des représentations purement formelles. Il s'agit là d'une distinction sans différence, puisque les représentations sont des contenus notionnels.

Pour que Fido ait une croyance il faut donc, selon la doctrine que je viens d'évoquer, qu'il ait une inscription, en « mode $C$ », d'une phrase de son système de représentations (i.e., en quelque sorte son « code cérébral»). Ceci revient simplement à dire, on l'a vu, que Fido doit être dans un état physique (token) qui joue dans sa «vie mentale » le type de rôle fonctionnel caractéristique de la croyance (ce type étant défini abstraitement par les principes de la psychologie populaire, en particulier la théorie de la décision). Il n'est ainsi pas nécessaire, pour établir si une inscription est (ou compte pour) une croyance d'en connaître le rôle propositionnel (c'est-à-dire de savoir de quelle représentation elle est une inscription), ni par 
conséquent le rôle fonctionnel spécifique. Il suffit de connaître le mode de ce rôle fonctionnel.

Cependant pour pouvoir dire qu'une inscription de Fido compte pour telle ou telle croyance, il faut en connaître le sens (et c'est évidemment à ce niveau que doit intervenir son rôle propositionnel). Cela ne veut pourtant pas dire qu'il faut pouvoir produire un énoncé qui ait le même sens que cette inscription, ni par conséquent qu'il faut être en mesure d'utiliser une attribution de croyance du type habituel (i.e., celui de (1)), qu'il s'agit précisément ici d'expliquer. Il suffit parfaitement de pouvoir dire, en termes métalinguistiques, en quoi consiste ce sens, c'est-à-dire à quelles conditions il est légitime pour un locuteur de prétendre reproduire ou exprimer ce sens à l'aide d'une phrase de sa propre langue 3 .

Je ne surprendrai probablement personne en me ralliant ici à la thèse traditionnelle (Fregéo-Husserlienne) selon laquelle la notion de sens (qu'il s'agisse du sens d'une inscription ou de celui d'une énonciation) comporte un double aspect, à savoir un aspect interne (subjectif, formel) et un aspect externe (objectif, causal). Il s'ensuit que les états intentionnels eux-mêmes ont un double aspect, ou peut-être faudrait-il dire que la notion naïve d'état intentionnel est hybride et recouvre en fait deux concepts distincts, qui répondent à des critères d'individuation distincts. Cette bifurcation s'explique, intuitivement, par le fait que les états intentionnels servent à régler les échanges entre la créature et son environnement : l'impact de l'environnement produit des stimuli, qui génèrent des croyances, qui par l'intermédiaire des désirs conduisent au comportement. Il est ainsi possible d'identifier un état intentionnel (d'un certain mode) soit par son contenu (i.e., le rôle propositionnel de l'inscription qui le réalise), soit par son objet (i.e., les conditions de vérité de cette inscription). Ce que je viens de dire là me semble

3. Cette remarque pourrait être moins anodine qu'elle ne paraît, dans la mesure où certains arguments tendant à montrer que les notions de croyance et de désir n'ont pas de place dans une psychologie scientifique semblent s'appuyer en grande partie, sinon exclusivement, sur les insuffisances (indéniables) du discours indirect ordinaire. Mais c'est une chose de savoir ce qu'est une croyance, c'en est une autre de savoir à quelles conditions on peut utiliser une attribution de croyance ordinaire pour la rapporter. Le fait que le discours indirect ne se prête pas à la formulation de généralisations psychologiques satisfaisantes ne montre pas, par lui-même, que les croyances et les désirs ne sont pas des entités honorables. 
relativement peu problématique, bien que plusieurs auteurs tendent à identifier les états intentionnels tantôt à leur contenu, tantôt à leur objet ${ }^{4}$.

Ce qui est plus problématique, c'est d'expliquer exactement quelles sont les relations entre le contenu et l'objet d'un état intentionnel, comment les attributions ordinaires d'états intentionnels peuvent être interprétées, et pourquoi elles semblent être plus volontiers interprétées comme les spécifications d'objet plutôt que de contenu. Il s'agit maintenant de chercher quelques éléments de réponse à ces questions, et de montrer que l'opposition contenu/ objet est motivée intuitivement.

\section{Questions d'individuation}

Il est facile de montrer que le contenu d'un état intentionnel (que j'identifierai ici au rôle propositionnel relatif de l'inscription qui le réalise, remettant à plus tard l'examen de la question de savoir s'il ne faudrait pas lui préférer le rôle propositionnel absolu) n'en détermine pas l'objet et ne saurait donc être confondu avec un sens fregéen. Je m'inspirerai pour le faire du type d'expériences de pensée popularisées par Putnam (1975a, 1982a, b). Le principe de

4. Ceci a d'ailleurs donné lieu à une controverse stérile autour de la thèse du solipsisme méthodologique (soutenue par Fodor 1980) selon laquelle la "psychologie cognitive » ne peut et ne doit formuler ses généralisations qu'en fonction de paramètres internes aux créatures, i.e. qu'elle doit faire abstraction des relations entre les créatures qu'elle étudie et l'environnement. La question disputée n'est pas tant de savoir si cette thèse est juste, ou fructueuse, que de savoir si comme le prétend Fodor cela autorise la psychologie cognitive à prétendre être une justification et une extension de la psychologie populaire. Fodor ne nie pourtant pas que deux inscriptions qui ont le même rôle propositionnel n'ont pas nécessairement le même objet, il refuse simplement d'individuer les états intentionnels par leur objet, et précend pouvoir expliquer les phénomènes qui l'intéressent en termes de contenus d'états intentionnels (dans ma terminologie). Cette querelle est sans objet si, comme je m'apprête à l'illustrer, les concepts ordinaires de croyance et de désir sont des concepts mixtes.

Quant au principe du solipsisme méthodologique proprement dit, il ne fait aucun doute qu'il y a place pour une discipline qui s'y conforme ; mais il est loin d'être évident qu'une telle discipline soit une psychologie. Elle risque fort d'être une neurophysiologie, s'il faut tenir compte quelque part de l'interaction créature/environnement pour pouvoir dire que tels ou tels états physiques d'une créature réalisent tels ou tels rôles fonctionnels ou propositionnels, i.e. pour déterminer quel est le système de représentations d'une créature. En fait, il semble que la réponse à cette question dépende de celle à la question de savoir si le même rôle fonctionnel doit pouvoir être réalisé dans plusieurs créatures, ou si chaque créature a son propre système de représentations. 
ces expériences consiste à imaginer deux créatures fonctionnellement identiques mais qui évoluent dans des environnements différents, et à guetter le murmure de l'intuition qui doit en principe nous dire si elles croient ou désirent la même chose. Il est bon toutefois d'insister sur le fait que je ne m'apprête pas à discuter l'interprétation des situations décrites par Putnam et Burge, puisque j'entends ne traiter ici que des états intentionnels supposés de créatures sublinguistiques, alors qu'ils n'ont considéré que le cas plus complexe des états intentionnels de sujets linguistiques.

On commencera par imaginer qu'une lointaine planète, la Terre-bis, est une réplique exacte de la Terre, «molécule pour molécule ». Il faut entendre par là, en particulier, que toute chose et toute créature terrestres ont sur la Terre-bis un (et un seul) double qualitativement indiscernable (i.e., indiscernable relativement à nos connaissances scientifiques), et ceci à tout moment de l'histoire de chacune de ces planètes. (Il se peut que cette supposition soit ultimement absurde, si on admet qu'il peut y avoir interaction causale entre la Terre et la Terre-bis, mais cela n'est pas préoccupant puisqu'elle n'est qu'un artifice de présentation dont on pourrait faire l'économie). Il s'ensuit que Fido a sur la Terre-bis un double, Fido-bis, qui non seulement a la même structure fonctionnelle que lui, mais réalise, à tout moment, exactement les mêmes états fonctionnels. En d'autres termes, Fido et Fido-bis mènent des vies qualitativement identiques; ils perçoivent les mêmes stimuli, produisent les mêmes réponses et passent par les mêmes suites d'états internes.

Supposons maintenant qu'à un certain moment Fido repère un chat et se lance à sa poursuite. Au même moment (c'est-à-dire à la même place dans la suite des états internes qu'ils ont en commun) Fido-bis voit un chat exactement semblable et le prend en chasse. Dans des circonstances favorables, un observateur pourrait alors vraisemblablement affirmer

(3) Fido croit qu'il poursuit un chat

et (4) Fido-bis croit qu'il poursuit un chat.

Par hypothèse, Fido et Fido-bis réalisent les mêmes états fonctionnels, qui ont par conséquent les mêmes rôles propositionnels relatifs. Mais s'il y a bien un sens dans lequel on peut dire ici qu'ils croient la même chose, il y a aussi un sens dans lequel ils ne croient manifestement pas la même chose puisque la croyance de Fido 
concerne Fido et celle de Fido-bis, Fido-bis; c'est-à-dire, leurs croyances respectives n'ont pas les mêmes conditions de vérité, mais elles ont le même contenu. Cette simple observation suffit à montrer que la notion naïve d'état intentionnel a bel et bien un double aspect; quelques remarques complémentaires sont peutêtre néanmoins utiles.

Les états intentionnels du type qu'on vient de considérer sont tout à fait fondamentaux. Ils se caractérisent, intuitivement, par leur réflexivité, qui se manifeste dans le fait qu'on ne pourrait apparemment en paraphraser le contenu qu'à la première personne du singulier (c'est-à-dire dans le fait que si Fido et Fido-bis parlaient français ils devraient tous les deux énoncer «Je poursuis un chat » pour dire ce qu'ils croient). Je les appellerai donc, à la suite de Lewis (1979), des états intentionnels de se, étant entendu qu'un état intentionnel est de se en vertu de son contenu et non de son objet. Leur importance particulière tient à la place privilégiée qu'ils paraissent devoir tenir dans les explications du comportement qui font intervenir des attributions d'état intentionnel. J'entends par là qu'aucun comportement ne paraît avoir d'explication complète (ou auto-suffisante) qui soit formulée uniquement en termes d'objets d'états intentionnels. Toute explication de ce genre doit quelque part invoquer ou présupposer des contenus d'états intentionnels, alors qu'une explication formulée uniquement en termes de contenus peut faire l'économie des objets (à condition que ce comportement soir lui-même décrit en termes solipsistes). Le principe du solipsisme méthodologique serait donc partiellement justifié. Il suffit d'un exemple trivial (et extrêmement schématique) pour l'illustrer.

Supposons que Fido se sente subitement affamé et décide de retourner à sa niche où l'attend un bol de nourriture; pour rejoindre sa niche il doit sauter la clôture du jardin de $\mathrm{M}$. Lambert. Il est facile d'expliquer, dans l'esprit de la psychologie populaire, pourquoi Fido a sauté cette clôture, à l'aide du raisonnement suivant :

(5a) Fido désirait retourner à sa niche Fido croyait que pour retourner à sa niche il devait sauter la clôture (de M. Lambert)

Fido a donc formé le désir de sauter cette clôture, et il l'a sautée. Il faudrait évidemment compléter ce raisonnement de diverses manières (en ajoutant, par exemple, que Fido se sentait capable de 
sauter la clôture, qu'au moment de sauter il n'avait pas de plus grand désir que celui de sauter, etc...). Mais je veux surtout faire remarquer qu'il n'a de plausibilité que sous la présupposition que les états intentionnels attribués à Fido sont de se. En d'autres termes, son efficacité (au moins apparente) ne lui vient pas du seul fait qu'il identifie les objets des états intentionnels de Fido. En effet le même raisonnement perd toute valeur explicative dès lors qu'on l'exprime sous une forme qui invite moins fortement à faire cette présupposition :

(5b) Fido désirait que Fido retourne à sa niche

Fido croyait que pour retourner à sa niche Fido devait sauter la clôture

Fido a donc formé le désir que Fido saute cette clôture, et il l'a sautée.

Manifestement, (5a) et (5b) attribuent à Fido des états intentionnels qui ont les mêmes conditions de vérité (ou dans la terminologie de Searle, les mêmes conditions de satisfaction), mais sous cette interprétation ils ne sont corrects ni l'un ni l'autre. La conclusion de (5b) ne suit des prémisses (non pas logiquement, mais en vertu des principes de la psychologie populaire qui lient les croyances et les désirs) que si on suppose que Fido croyait, de plus, qu'il était luimême Fido, et si cette supposition porte sur le contenu de la croyance ainsi attribuée à Fido. On s'en rendra peut-être compte plus facilement en transposant ces deux raisonnements sous une forme auto-attributive :

(6a) Je désire retourner à ma niche

Je crois que pour retourner à ma niche je dois sauter la clôture

Il faut donc que je saute la clôture

(6b) Je désire que Fido retourne à sa niche

Je crois que pour retourner à sa niche Fido doit sauter la clôture

Il faut donc que Fido saute la clôture

On ne voit évidemment pas pourquoi, en (6b), le fait de conclure que Fido doit sauter la clôture devrait amener ce dernier à la sauter, s'il ne s'identifie pas à Fido (i.e., s'il ne croit pas que "Fido" le désigne lui). La morale de cette fable est que le lien établi par la psychologie populaire entre les comportements et les états intentionnels repose sur la possibilité d'individuer les états intentionnels 
par leur contenu (et leur mode), conformément au principe du solipsisme méthodologique. Cela ne signifie pas qu'il soit toujours suffisant, pour expliquer un comportement selon les schémas de la psychologie populaire, d'invoquer des contenus d'états intentionneis. Tout dépend de la façon dont le comportement à expliquer est décrit. En l'occurrence, ni (5a) ni (6a) n'expliquent pourquoi Fido a effectivement sauté la clôture ; ils expliquent tout au plus pourquoi il a essayé de la sauter, c'est-à-dire pourquoi certaines terminaisons nerveuses ont provoqué les mouvements corporels qui l'ont finalement propulsé de l'autre côté de la clôture. Mais aussitôt que l'explanandum est décrit en termes non solipsistes (comme c'est généralement le cas) il faut aussi faire intervenir dans l'explication les relations causales entre la créature et son environnement. Il n'en reste pas moins qu'une explication solipsiste d'un comportement, bien qu'elle soit rarement recherchée par la psychologie populaire, peut avoir une certaine autonomie qu'une explication non-solipsiste n'a pas (tant qu'elle est formulée en termes d'attributions d'états intentionnels, i.e. tant qu'elle n'est pas purement naturaliste).

Le fait qu'une créature capable d'états intentionnels doive apparemment être aussi capable d'états intentionnels de se soulève cependant une difficulté pour l'idée selon laquelle un état intentionnel consiste (en partie) dans le fait d'avoir une représentation d'un état de choses. Cela semble en effet impliquer que le système de représentations de toute créature doit contenir sinon un équivalent du pronom « je» tel qu'il est utilisé dans une langue publique, du moins un élément qui y joue un rôle analogue à un emploi du pronom « je » par un locuteur (du français) donné, ou à une sorte de nom propre « originaire » de soi-même. Mais une telle conséquence serait très gênante, dans la mesure où elle reviendrait à toutes fins pratiques à concéder une forme de conscience de soi à toute créature capable d'états intentionnels. On pourrait éventuellement accepter cette conséquence, et admettre que les attributions d'états intentionnels de se à des créatures sublinguistiques ne sont jamais littéralement vraies, et témoignent d'une attitude anthropocentrique (ce qui est sans doute le cas en dernière analyse). Une autre solution serait d'affaiblir la doctrine des représentations mentales et d'accorder un statut particulier aux états intentionnels de se, en admettant qu'ils n'impliquent pas la réalisation de représentations «propositionnelles ». 
Il faut se rappeler que les états intentionnels sont des dispositions conditionneiles au comportement et qu'ils ne sont attribués aux créatures sublinguistiques que sur la base de leur comportement, dans le but de le « rationaliser ». Peut-être le simple fait qu'un état intentionnel soit réalisé dans telle créature et contribue par conséquent à expliquer le comportement de cette créature est-il suffisant pour qu'il puisse être considéré comme de se. Je suis plutôt enclin à penser que tous les états intentionnels (singuliers, tout au moins) peuvent être considérés comme de se, mais que certains seulement sont «irréductiblement » de se; ou peut-être devrais-je dire qu'il m'apparaît utile, pour flatter le sens commun, de distinguer deux notions d'état intentionnel. Car en effet l'idée que tous les états intentionnels sont de se heurte le sens commun dans la mesure où elle implique que les états intentionnels de deux créatures distinctes ne peuvent avoir les mêmes conditions de vérité. Cette idée est quand même assez plausible, au moins dans le cas des états intentionnels singuliers, puisqu'il est difficile d'imaginer comment une créature pourrait identifier un particulier autrement que par ses relations (spatio-temporelles) avec elle-même.

Supposons que Fido-bis soit miraculeusement transporté sur la Terre et qu'il se lance à la poursuite du même chat que Fido. Le chat se réfugie dans un arbre au pied duquel les deux chiens aboient furieusement (et stupidement). Certes on ne peut plus dans ces conditions continuer à supposer que Fido et Fido-bis réalisent exactement les mêmes états fonctionnels, puisqu'ils peuvent maintenant interagir et ne reçoivent forcément plus les mêmes stimuli (car ils s'insèrent désormais dans le même environnement). On admettra toutefois (au moins pour les besoins de la discussion) qu'ils restent suffisamment semblables (fonctionnellement) pour qu'on puisse supposer qu'ils croient tous les deux qu'il y a un chat dans cet arbre, et que leurs croyances respectives ont le même contenu (à toutes fins utiles). La question est de savoir comment se présente ce contenu, et en particulier cette partie du contenu, qu'on peut appeler (peut-être un peu paradoxalement) le "contenu référentiel », qui sert à identifier les particuliers sur lesquels portent leurs croyances respectives. Il y a à première vue deux possibilités, qui consistent à paraphraser ce contenu sous l'une ou l'autre des deux formes suivantes:

(7) il y a un chat dans l'arbre devant moi

(8) il y a un chat dans $n$ 
où « $\mathrm{n}$ » est un terme singulier «directement référentiel » qui dénote l'arbre qui est devant Fido et Fido-bis. Si on suppose que le contenu des états intentionnels de Fido et de Fido-bis correspond à (7) alors manifestement ces états intentionnels sont de se et n'ont pas les mêmes conditions de vérité, bien qu'ils aient en quelque sorte une partie de leurs conditions de vérité en commun (dont les conditions de vérité de (8) font à leur tour partie). Si au contraire on choisit (8), alors les états intentionnels en question ont non seulement le même contenu, mais aussi le même objet, comme le souhaite apparemment le sens commun, qui semble ici opérer avec une notion d'état intentionnelle partiel ou « incomplet ». On pourrait être ici tenté de parler d'état intentionnel de re plutôt que d'état intentionnel partiel, et cela ne serait pas tout à fait sans fondement ; mais il faut remarquer que l'état intentionnel dont (7) pourrait exprimer le contenu est de re aussi bien que celui correspondant à (8) puisqu'il contient lui aussi un élément directement référentiel (à savoir «moi »).

Quoi qu'il en soit il importe peu pour mon propos qu'on accepte ou non l'idée que les états intentionnels qui ne sont pas de se sont en quelque sorte incomplets, ou même celle qu'une créature puisse avoir des états intentionnels de se. Il suffit de remarquer que même si on admet que le système de représentations mentales d'une créature sublinguistique puisse contenir des termes singuliers primitifs plus ou moins assimilables à des noms propres logiques "russelliens» (qui pourraient par exemple être réalisés sous la forme de perceptions) il n'en reste pas moins vrai que deux créatures peuvent réaliser des états intentionnels qui ont le même contenu (par exemple celui de (8)) sans avoir le même objet (par exemple, si Fido-bis est sur la Terre-bis, devant un arbre exactement semblable à celui devant lequel se trouve Fido, etc...).

Les remarques qui précèdent ne tiennent compte que de l'aspect référentiel des états intentionnels, dont il est difficile de ne pas croire qu'ils ont aussi un aspect conceptuel (ou prédicatif). Cela soulève la question de savoir si deux états intentionnels qui ont le même contenu conceptuel doivent avoir le même objet conceptuel, c'est-à-dire, si le rôle conceptuel d'un terme général (du système de représentations d'une créature) en détermine l'extension. On sait que les expériences de pensée discutées par Putnam et Burge ont été interprétées (par eux du moins) comme apportant une réponse 
négative à cette question. Il semble cependant que la plausibilité de ce verdict soit étroitement liée à l'hypothèse que les créatures à qui on attribue des états intentionnels sont des sujets linguistiques (et même dans ce cas le verdict reste extrêmement douteux).

Retournons donc sur la Terre-bis, et supposons cette fois que la créature poursuivie par Fido-bis n'est pas vraiment un chat, mais une mécanique destinée à le tromper, et qui a toutes les apparences et les réactions d'un chat. Il s'agit alors de savoir si Fido et Fido-bis croient tous les deux que c'est un chat qu'ils poursuivent. Je rappelle que par hypothèse, Fido et Fido-bis réalisent les mêmes rôles fonctionnels et que par conséquent leurs états intentionnels respectifs ont les mêmes rôles conceptuels. Il faut ici distinguer deux questions: i) les objets de croyance de Fido et de Fido-bis peuvent-ils être caractérisés à l'aide du même terme général?, et ii) peut-on utiliser le terme de «chat » pour caractériser l'objet conceptuel soit de la croyance de Fido, soit de celle de Fido-bis, soit de celles des deux?

Il semble indéniable que dans les circonstances que je viens d'évoquer ni Fido ni Fido-bis ne font une erreur en appliquant leur contenu prédicatif commun (i.e., leur «concept») aux créatures qu'ils poursuivent. Par conséquent l'extension de ce concept est la même dans la tête de Fido ou dans celle de Fido-bis, et il doit être légitime d'utiliser un seul et même terme général pour caractériser à la fois l'objet et le contenu conceptuels de leurs états intentionnels respectifs, à condition bien sûr de disposer d'un terme approprié. Or il est clair que si un non-chat tombe sous un certain concept, ce concept ne peut être le concept de chat. Ainsi ni Fido ni Fido-bis n'ont notre concept de chat, bien qu'ils aient tous deux développé leur concept commun à la suite d'interactions causales avec de vrais chats; et l'emploi du terme de «chat» pour spécifier l'objet ou le contenu conceptuels de leurs croyances respectives ne peut donc être qu'approximatif.

Ce jugement n'implique nullement qu'il soit impossible à une créature sublinguistique de se tromper en appliquant un concept à un particulier. Mais une erreur doit être décelable ; c'est-à-dire, consıste dans le fait qu'un stimulus anticipé pourrait ne pas se réaliser (ou qu'un désir pourrait être frustré). Or cette possibilité est exclue a priori dans la situation envisagée. Même si on supposait 
que le pseudo-chat poursuivi par Fido-bis n'est pas parfaitement indiscernable d'un vrai chat (en ceci, par exemple, qu'il ne saignerait pas s'il était blessé), Fido-bis ne ferait une erreur en croyant poursuivre une créature qui tombe sous son concept de «quasichat » que si ce concept incluait la condition que les objets auxquels il s'applique doivent avoir certains traits que ce pseudo-chat n'a pas (par exemple, qu'ils doivent saigner quand ils sont blessés). Mais il est possible que le concept de Fido-bis n'ait pas cette caractéristique (par exemple, s'il n'a jamais vu un chat blessé), et que là non plus il ne soit pas dans l'erreur.

Supposons maintenant qu'il n'y ait jamais vraiment eu de chats sur la Terre-bis, mais des créatures (naturelles ou artificielles) parfaitement indiscernables (pour les chiens) de chats terrestres. Ainsi chaque fois que Fido rencontre un chat, Fido-bis rencontre un chat-bis exactement semblable, qui réagit de la même façon, etc... Même dans ces conditions, où Fido-bis n'a jamais été en contact avec de vrais chats, il reste légitime de dire que Fido et Fido-bis ont le même concept (qui n'est toujours pas le concept de chat). On pourrait supposer, pour s'en convaincre, qu'un chat soit expédié sur la Terre-bis et mis en présence de Fido-bis. Si le concept de ce dernier avait une extension différente de celui de Fido, il faudrait dire qu'il est dans l'erreur en croyant être en face d'une créature qui tombe sous ce concept, alors que Fido lui (qui est aussi en présence d'un chat) ne le serait pas. Mais il n'y a aucune raison de penser que Fido-bis se trompe, puisque par hypothèse aucune de ses attentes concernant cette créature (i.e., celle qu'il a en vertu de l'application de son concept de quasi-chat) ne peut être déçue. Par conséquent s'il n'est pas légitime de dire de Fido-bis qu'il croit poursuivre un chat cela ne l'est pas non plus de le dire de Fido (puisque leurs concepts respectifs ont la même extension). Ainsi, contrairement à ce qu'une lecture rapide de Putnam et Burge pourrait laisser croire, ce n'est pas parce qu'une créature a eu affaire à des chats qu'elle a pour autant un concept de chat. Je crois qu'il serait possible de montrer qu'il lui faut aussi avoir le concept de réalité (et donc d'erreur) et que cela revient à dire qu'il lui faut être un sujet linguistique et par conséquent être en mesure d'attribuer des états intentionnels à autrui. D'une certaine façon je n'ai fait ici que déblayer le terrain en vue d'établir éventuellement cette conclusion. 
De la discussion qui précède, on peut retenir que le contenu d'un état intentionnel (d'une créature sublinguistique, si une telle créature peut avoir des états intentionnels) n'en détermine pas l'objet, et que cela tient uniquement aux caractéristiques particulières des contenus référentiels, à l'exclusion des contenus conceptuels. Il resterait, pour en finir avec la question des relations entre le contenu et l'objet des états intentionnels à se demander s'il est possible que deux états intentionnels qui ont le même objet n'aient pas le même contenu. On concèdera facilement (et il est traditionnel d'admettre) que c'est tout à fait possible, du moins s'il s'agit de deux états intentionnels d'une même créature, puisqu'une créature peut vraisemblablement appréhender les mêmes particuliers sous différents aspects, et disposer de contenus conceptuels distincts mais en fait coextensifs. Il n'en va cependant plus de même, trivialement, dans le cas d'états intentionnels de créatures distinctes, s'il se confirme que deux créatures ne peuvent jamais réaliser des états intentionnels singuliers ayant exactement le même objet.

\section{Questions d'interprétation}

Le moment semble donc venu de se demander, à la lumière des remarques qui précèdent, comment interpréter les attributions ordinaires (i.e., en discours indirect) d'états intentionnels à des créatures sublinguistiques, et en particulier si et à quelles conditions il est légitime de considérer qu'elles affirment que la créature concernée a une inscription qui a le même sens que celui dans lequel la phrase complétive est utilisée par le locuteur. Si cela devait être littéralement et systématiquement vrai, il faudrait considérer comme fausse toute attribution (indirecte) d'état intentionnel dont la complétive n'aurait pas (en contexte) à la fois le même contenu et le même objet qu'une inscription de la créature visée. Or le simple fait que le contenu d'un état intentionnel n'en détermine pas l'objet, ni l'objet le contenu, exclut qu'il soit toujours possible de reproduire à la fois le contenu et l'objet d'un état intentionnel à l'aide d'un seul énoncé. D'un autre côté le fait que les états intentionnels ont un double aspect (si c'est un fait) semble impliquer que les attributions d'états intentionnels se prêtent à deux types d'interprétations, que je qualifierai de « solipsiste » et «non solipsiste», respectivement. Il semble que de façon générale une attribution d'état intentionnel vise d'abord à en spécifier l'objet, au détriment du contenu. Cela 
pourrait s'expliquer aussi bien par le fait que cette forme d'expression ne permet pas toujours de reproduire exactement le contenu de l'état intentionnel en question, que par le fait qu'en énonçant une phrase de la forme « $x$ croit que $p$ », le locuteur utilise « $\mathrm{p}$ » et qu'en principe on utilise une phrase pour décrire un fragment du monde extérieur et non pour en exhiber le rôle propositionnel. Ce ne sont évidemment là que des impressions générales que la discussion qui suit est destinée à expliciter.

Ainsi, une énonciation de

(9) Fido croit qu'il y a un chat dans l'arbre devant lui devrait en principe pouvoir être interprétée de l'une ou l'autre des deux façons suivantes :

(10) Fido a une inscription en mode $C$ qui a le même contenu que «il y a un chat dans l'arbre devant lui » en tant que phrase de ma langue énoncée par moi maintenant.

(11) Fido a une inscription en mode $C$ qui a le même objet que «il y a un chat dans l'arbre devant lui » en tant que phrase de ma langue énoncée par moi maintenant.

Je n'examinerai ici que le cas où «en tant que phrase de ma langue énoncée par moi maintenant » renvoie au sens (contenu ou objet) littéral quaurait la phrase en question dans l'idiolecte du locuteur (i.e., dans ce qu'il considère comme la langue de sa communauté linguistique) s'il devait l'énoncer à ce moment. Je prétends ainsi ignorer (provisoirement) les complications qui pourraient venir de ce que le locuteur prête à la phrase en question un sens (contenu ou objet) non littéral, ou de ce qu'il vise le sens que cette phrase a «réellement» dans la langue (i.e., pour sa communauté linguistique) et sur lequel il reconnaît qu'il pourrait se tromper. Je supposerai aussi, jusqu'à nouvel ordre, que la description «l'arbre devant lui » dans (9) est dans la portée du verbe d'attitude propositionnelle.

On notera d'abord que (10) ne semble correspondre à une interprétation possible de (9) que si le pronom « lui » y fonctionne comme un démonstratif. En d'autres termes, si Fido pouvait avoir une inscription en mode $C$ d'une phrase telle que « il y a un chat dans l'arbre devant lui», on ne serait pas tenté de dire qu'il croit être lui-même devant un arbre où se cache un chat, même s'il 
s'avérait que cette occurrence de «lui » renvoie en fait à Fido luimême, de sorte qu'on puisse dire que Fido croit de Fido qu'il est devant un arbre où se cache un chat. Il serait certes un peu bizarre, mais pas incorrect, de décrire une telle situation, en style indirect, à l'aide de (9); il s'agit donc apparemment d'une interprétation légitime de (9).

Cependant l'interprétation la plus naturelle de (9) (hors contexte) est sans doute celle qui fait de «lui» un pronom anaphoriquement lié à « Fido ». Mais cette interprétation n'identifie explicitement que l'objet de la croyance de Fido et ne dit rien de son contenu. En effet le pronom «lui » occupe alors une position référentielle, et on pourrait lui substituer n'importe quel terme directement référentiel qui dénote Fido sans affecter les conditions de vérité de l'énoncé qui en résulterait.

Pour interpréter (9) comme une identification du contenu de la croyance de Fido, il faut apparemment considérer le pronom « lui » dans (9) comme un réflexif indirect (ou dans la terminologie de Castañeda 1967, comme un quasi-indicateur), c'est-à-dire qu'il faut l'interpréter comme une façon de signaler un état intentionnel de se. Autrement dit, si le pronom «lui» dans (9) doit être interprété comme une contribution à l'identification du contenu de l'état intentionnel attribué (et s'il n'est pas utilisé comme un démonstratif), il faut comprendre (9) dans le sens de :

(12) Fido a une inscription en mode $C$ dont le contenu est identique à celui qu'aurait dans mon présent idiolecte une énonciation littérale par moi maintenant de « il y a un chat dans l'arbre devant moi »

Peut-être jugerait-on plus naturelle la formulation suivante, qui est pourtant équivalente, puisque le contenu d'une énonciation n'est pas fonction des circonstances d'énonciation (i.e., le temps et le lieu, et l'identité du locuteur):

(13) Fido a une inscription en mode $C$ dont le contenu est identique à celui qu'aurait dans mon présent idiolecte une énonciation littérale par lui maintenant de «il y a un chat dans l'arbre devant moi ».

Si (13) paraît plus naturelle que (12), en tant qu'interprétation de (9), c'est sans doute parce qu'elle contient aussi l'information suffisante (contrairement à (12)) pour assigner à lá croyance de 
Fido un objet approprié. Mais s'il s'agit d'identifier l'objet de la croyance de Fido, il y a une façon plus directe et plus explicite de le faire, comme on le verra plus loin. On peut sans doute voir dans cet ordre de préférence une indication du fait que la fonction première d'une attribution d'état intentionnel est d'en identifier l'objet et non le contenu, et qu'il n'est nécessaire de voir dans une attribution d'état intentionnel une identification du contenu que dans certains cas particuliers (par exemple, quand l'interprétation non solipsiste n'est pas possible, ou n'est pas acceptable). On notera qu'il n'est possible de remonter, pour ainsi dire, de la complétive utilisée au contenu de l'état intentionnel attribué que lorsqu'elle contient des éléments qui peuvent être interprétés comme des quasi-indicateurs. Il est bon de souligner que ce n'est pas le cas des déictiques purs en contexte indirect. Ainsi un énoncé tel que :

(14) Fido croit qu'il y a un chat dans l'arbre devant moi n'a apparemment pas d'interprétation purement solipsiste complète, bien que la distance ne soit pas plus grande entre (14) et

(15) Fido a une inscription en mode $\mathrm{C}$ dont le contenu est identique à celui qu'aurait dans mon présent idiolecte une énonciation littérale par moi maintenant de « il y a un chat dans l'arbre devant lui »

qu'entre (9) et (12). Le fait est qu'en utilisant «moi » le locuteur qui énonce (14) ne donne aucun indice quant à la façon dont il se présente à Fido, alors que dans (9) l'emploi de «lui » peut être interprété comme l'indice d'un état intentionnel de se.

On peut en fait douter qu'il y ait des attributions d'état intentionnel singulier qui admettent une interprétation solipsiste complète et qui ne contiennent pas de quasi-indicateurs. On retrouve ici, sous un autre angle, la question de savoir si une créature peut avoir des états intentionnels singuliers dont le contenu ne soit pas de se. Comme une attribution d'état intentionnel qui utiliserait une description définie (complète) purement qualitative (i.e., qui ne contiendrait ni déictiques ni quasi-indicateurs) ne serait pas une attribution d'état intentionnel singulier, il n'y a qu'un cas à considérer, celui où les seuls termes singuliers utilisés sont des noms propres, comme par exemple dans :

(16) Fido croit que Félix est un chat.

La question est de savoir si cet énoncé peut vouloir dire que 
(17) Fido a une inscription en mode $C$ dont le contenu est identique à celui qu'aurait dans mon présent idiolecte une énonciation littérale par moi maintenant de « Félix est un chat ».

Il semble que oui, mais seulement si «Félix » fonctionne dans l'idiolecte du locuteur comme un nom propre logique et si le système de représentations de Fido contient de tels noms. Or dans ce cas «Félix» ne contribue en rien à déterminer le contenu de "Félix est un chat» puisque tous les noms propres logiques ont pour ainsi dire le même contenu (c'est-à-dire, «Fido est un chat » et «Félix est un chat» auraient dans cette hypothèse la même «syntaxe logique»). On obtiendrait donc ainsi une interprétation solipsiste «incomplète» de (16), qui ne rendrait compte que du contenu conceptuel de l'état intentionnel de Fido. Si «Félix» fonctionne au contraire comme une description définie identifiante (qui ne soit pas purement qualitative) alors on retombe sur un des cas déjà examinés, selon que cette description contient des déictiques purs ou des quasi-indicateurs (sauf si on considère que cette description n'est pas dans la portée du verbe «croire»).

Il est temps de se rappeler que j'ai choisi plus haut d'identifier l'aspect interne du sens d'une inscription à son contenu relatif. Or la formulation (12) fait allusion au contenu d'une énonciation d'une phrase d'une langue publique (ou plus exactement à ce que le locuteur considère comme la langue de sa communauté linguistique). Le contenu relatif d'un état intentionnel est fonction de son contenu absolu et de ses relations causales (potentielles) aux autres états intentionnels du même mode qui sont réalisés dans la créature donnée (les relations aux états intentionnels d'autres modes sont exclues parce qu'elles ne sont pas uniquement fonction de leur contenu, mais aussi de leur mode). De façon analogue, le contenu relatif d'une énonciation d'une phrase pourrait être fonction de son contenu absolu (i.e., du rôle inférentiel de la phrase énoncée dans l'idiolecte en question) et des présuppositions du locuteur au moment de l'énonciation (i.e. l'information d'arrière-plan qu'il prend pour acquise). Il semblerait dans ces conditions qu'un locuteur ne puisse identifier le contenu relatif d'un état intentionnel qu'à condition de connaître (et de présupposer connu) celui de tous les états intentionnels du même mode qui sont réalisés dans la créature donnée et auxquels il est causalement lié. Cela suggère par 
la même occasion qu'un état intentionnel d'une créature ne peut avoir le même contenu relatif qu'un état intentionnel d'une autre créature que si tous ses états intentionnels ont le même contenu relatif qu'un état intentionnel de cette autre créature. Le caractère holistique des contenus d'états intentionnels semble donc exclure, à toutes fins pratiques, qu'on puisse identifier le contenu d'un état intentionnel isolé.

Je ne crois pas qu'on puisse gagner quoi que ce soit à remplacer la notion de contenu relatif par celle de contenu absolu. En effet, s'il semble excessif d'exiger que deux créatures réalisent exactement la même configuration d'états fonctionnels pour pouvoir dire que deux d'entre eux ont le même contenu, il serait probablement insuffisant de se contenter de la condition qu'elles aient toutes les deux une inscription de la même représentation (qui ait par conséquent le même contenu absolu). Il faut dire que la notion de contenu absolu est extrêmement pauvre, et que le contenu absolu d'une représentation se confond vraisemblablement avec sa forme logique (puisqu' il est en quelque sorte totalement a priori; l'hypothèse contraire reviendrait à admettre qu'une créature dispose de concepts empiriques innés. Il est vrai que Fodor 1975 n'hésite pas à suggérer que tous les concepts sont innés!). D'autre part la notion de contenu absolu n'est pas moins holistique que celle de contenu relatif puisque deux inscriptions n'ont le même contenu absolu que si elles réalisent des représentations relevant du même système.

Il apparaît donc qu'une attribution d'état intentionnel à une créature ne peut généralement pas être interprétée comme une affirmation à l'effet qu'une inscription de cette créature a le même contenu relatif (ou absolu) que la complétive utilisée. Il serait encore insuffisant de dire (à la suite de Stich 1982, 1983) que le contenu relatif doit être semblable (ou partiellement identique) à celui de la complétive utilisée puisque cela aurait pratiquement l'effet inverse, de rendre vraies (presque) toutes les attributions d'état intentionnel. En effet comme deux inscriptions qui ont le même contenu absolu ont forcément des contenus relatifs semblables, il suffirait, par exemple, que le contenu absolu de l'état intentionnel visé soit identique (ou même semblable) à celui de la complétive utilisée (ou plus exactement de la phrase obtenue en remplaçant les quasi-indicateurs par des déictiques appropriés) pour qu'une 
attribution d'état intentionnel doive être comptée pour vraie. Il faut donc apparemment reconnaître que la ressemblance doit atteindre un degré suffisant, c'est-à-dire dépasser un certain seuil, et que ce seuil varie en fonction du contexte et des intérêts du locuteur. Cette variabilité est sans doute liée au fait que les attributions d'états intentionnels à des créatures sublinguistiques visent, typiquement, à en expliquer le comportement selon les schémas d'une théorie psychologique rudimentaire (qui fait partie des présuppositions du locuteur). Il est vraisemblable que le degré de ressemblance requis soit précisément le degré de ressemblance nécessaire pour que l'attribution en question contribue à expliquer ou rationaliser le comportement visé (relativement à la théorie présupposée et aux présuppositions «auxiliaires » portant sur les autres états intentionnels réalisés dans la créature concernée). Ceci permettrait de suggérer que la formulation suivante constitue une meilleure approximation que (12) de l'interprétation solipsiste de (9) :

(18) Fido a une inscription en mode $C$ dont le contenu relatif est suffisamment semblable à celui qu'aurait dans mon présent idiolecte une énonciation littérale par moi maintenant de «il y a un chat dans l'arbre devant moi ».

Mais il se pourrait bien, malgré tout, que le repli que constitue le passage de (12) à (18) soit un peu prématuré et surtout inutile. Je ne suis pas sûr en effet qu'on doive s'alarmer d'interpréter les attributions indirectes d'états intentionnels de telle manière qu'elles soient probablement toutes fausses (si on retient la notion d'identité) ou toutes vraies (si on retient la notion de ressemblance, sans seuil d'acceptabilité). On pourrait ainsi retenir (12) comme énoncé des conditions de vérité de l'interprétation solipsiste d'une énonciation de (9), en abandonnant à une éventuelle « théorie de la conversation » le problème d'expliquer comment de tels énoncés peuvent parfois être jugés acceptables. On verra que dans le cas de l'interprétation non solipsiste une telle attitude permettrait une simplification appréciable.

Je me tourne donc maintenant vers l'interprétation non solipsiste des attributions d'états intentionnels, selon laquelle elles visent à identifier l'objet des états intentionnels attribués. Il convient d'abord de souligner que le caractère holistique du contenu d'un état intentionnel en contamine aussi (en partie) l'objet dans 
la mesure où (comme j'ai tenté de le montrer plus haut) le contenu conceptuel d'un état intentionnel en détermine l'objet conceptuel. (Sans doute faudrait-il aussi, par conséquent, distinguer un objet conceptuel relatif et un objet conceptuel absolu, qu'on pourrait identifier, respectivement, à la propriété déterminée par le contenu conceptuel relatif et à celle déterminée par le contenu conceptuel absolu). Il en résulte apparemment qu'il faut encore faire appel à la relation de ressemblance (cette fois entre objets) même quand il s'agit de fournir une interprétation non solipsiste d'une attribution d'état intentionnel (si toutefois on retient cette solution dans le cas de l'interprétation solipsiste).

Il n'est certes pas nécessaire, en principe, de reproduire le contenu conceptuel d'un état intentionnel pour en identifier l'objet conceptuel (puisqu'il suffit d'utiliser un énoncé dont le contenu conceptuel détermine la même propriété), mais il semble que la seule garantie qu'on puisse avoir d'avoir correctement identifié un objet conceptuel vienne de la présomption d'avoir produit un énoncé qui a le même contenu conceptuel. A fortiori, on ne peut croire que l'objet conceptuel de l'énoncé produit ressemble à celui de l'état intentionnel visé que si on peut croire que son contenu conceptuel ressemble suffisamment à celui de cet état intentionnel. C'est peut-être pour cette raison qu'on a apparemment tendance à interpréter la composante prédicative de la complétive utilisée pour attribuer un état intentionnel comme si elle visait à révéler (mimer, exhiber) le contenu (relatif) de cet état intentionnel, ou plus exactement à ne guère faire la différence à ce niveau entre une lecture solipsiste et une lecture non solipsiste (puisque identifier le contenu conceptuel semble être la meilleure façon d'identifier l'objet conceptuel). Il est permis en tout cas d'y voir une raison supplémentaire pour faire intervenir la relation de ressemblance dans l'interprétation non-solipsiste, et donc pour remplacer (11) par quelque chose comme:

(19) Fido a une inscription en mode $C$ dont l'objet (relatif) ressemble suffisamment à celui qu'aurait dans mon présent idiolecte une énonciation littérale par moi maintenant de «il y a un chat dans l'arbre devant lui».

Cela soulève immédiatement deux difficultés. Premièrement, cette formulation paraît trop libérale, dans la mesure où il est loin d'être clair qu'en ce qui concerne les objets référentiels des états 
intentionnels il soit encore acceptable de renoncer à parler d'identité pour se contenter de ressemblance suffisante. Autrement dit, il est difficilement concevable qu'on puisse dire, par exemple, que Fido croit que Félix est un chat, si l'état intentionnel de Fido est causalement relié à quelque chose qui n'est pas identique à Félix, quel que soit le degré de ressemblance qu'il puisse y avoir entre Félix et cette chose.

Deuxièmement, cette formulation ne suffit à identifier ni l'objet référentiel de l'état intentionnel en question, ni même quelque chose qui y ressemble, pour la bonne raison qu'elle ne contient pas l'information suffisante sur le contexte de l'énonciation envisagée pour déterminer la dénotation du pronom «lui » (ni par conséquent les conditions de vérité qui seraient celles d'une telle énonciation). On notera toutefois à cet égard que (14), qui au lieu de «lui» contient le déictique pur «moi», paraît admettre une interprétation de la forme de (19) qui évite cette difficulté :

(20) Fido a une inscription en mode $C$ dont l'objet (relatif) ressemble suffisamment à celui qu'aurait dans mon présent idiolecte une énonciation littérale par moi maintenant de «il y a un chat dans l'arbre devant moi».

C'est que la dénotation des déictiques purs est déterminée par les conditions de l'énonciation envisagée.

Supposons que le pronom «lui» fonctionne dans (9) comme un démonstratif (en conservant toujours l'hypothèse que «l'arbre devant lui » est dans la portée du verbe «croire »). Un démonstratif n'a de dénotation que lorsqu'il est accompagné d'une «démonstration» (dans la terminologie de Kaplan 1977) par le locuteur de ce dont il veut parler. À supposer donc qu'en énonçant (9) le locuteur indique démonstrativement $M$. Lambert, il semble qu'on pourrait rendre compte de cet énoncé en complétant (19) à l'aide des mots «si au moment de prononcer "lui" j'indiquais démonstrativement $\mathrm{M}$. Lambert », où la position occupée par «M. Lambert » est transparente, et pourrait donc être occupée, notamment, par une occurrence du pronom « lui », accompagnée d'une démonstration de $\mathrm{M}$. Lambert. On obtiendrait un résultat équivalent si on acceptait de substituer «M. Lambert» (ou tout autre terme directement référentiel qui dénote $M$. Lambert indépendamment du contexte) à «lui» dans «il y a un chat dans l'arbre devant lui », puisque le pronom « lui » contribue aux conditions de vérité d'une énonciation 
de cette phrase exclusivement en vertu de sa dénotation. De la même façon, dans le cas où ce pronom serait dans (9) en relation anaphorique à «Fido», il suffirait apparemment de substituer «Fido » à « lui » pour obtenir l'interprétation désirée; on pourrait aussi se contenter de compléter (19) avec les mots «si j'utilisais "lui" pour désigner Fido ».

La procédure qui consiste à compléter (19) de la façon appropriée est d'application plus générale. Supposons en effet qu'au lieu de (9) le locuteur énonce

(21) Le chien de M. Lambert croit qu'il y a un chat dans l'arbre devant lui,

où « lui » est en relation anaphorique à « le chien de M. Lambert ». Il serait certainement incorrect du substituer «le chien de M. Lambert » à «lui» dans la complétive mentionnée par (19), mais non de compléter (19) avec les mots «si j'utilisais "lui" pour désigner le chien de $M$. Lambert ». On pourrait certes envisager de remplacer « lui » par « Fido » dans la complétive, mais rien ne nous assure que le locuteur sache que le chien de M. Lambert s'appelle «Fido ».

Le cas où la description « l'arbre devant lui » n'est pas dans la portée du verbe «croire» soulève aussi la même difficulté, à laquelle s'en ajoute une autre, qui vient de l'obligation dans laquelle on se trouve alors d'envisager l'énonciation d'une phrase où cette description ne figurerait pas. On pourrait certes avoir recours à une formulation telle que :

(22) Fido a une inscription en mode $C$ dont l'objet (relatif) ressemble suffisamment à celui qu'aurait dans mon présent idiolecte une énonciation littérale par moi maintenant de «il y a un chat dans ceci» si en prononçant «ceci » j'indiquais démonstrativement l'arbre devant lui.

Il semble donc qu'on n'ait d'autre ressource, en fin de compte, que de spécifier séparément l'objet conceptuel et le ou les objets référentiels de l'état intentionnel attribué. Le résultat n'est pas très élégant, mais a tout de même l'avantage d'éviter la première difficulté mentionnée plus haut :

(23) Fido a une inscription en mode $C$ dont l'objet conceptuel (relatif) ressemble suffisamment à celui qu'aurait dans 
mon présent idiolecte une énonciation littérale par moi maintenant d'une phrase de la forme « il y a un chat dans l'arbre devant — (ou M. Lambert)

(24) Fido a une inscription en mode C dont l'objet conceptuel (relatif) ressemble suffisamment à celui qu'aurait dans mon présent idiolecte une énonciation littérale par moi maintenant d'une phrase de la forme «il y a un chat dans - , et dont l'objet référentiel est l'arbre devant Fido (ou M. Lambert).

Ceci paraît rendre compte des quatre interprétations non solipsistes de (9) évoquées précédemment. On rejoint ainsi tout à fait la doctrine traditionnelle selon laquelle les états intentionnels singuliers sont des relations entre les créatures où elles sont réalisées et des états de choses, c'est-à-dire des complexes de particuliers et de propriétés. Il est peut-être bon de souligner que dans le cas des états intentionnels de créatures sublinguistiques la relation entre une inscription et ses objets référentiels peut vraisemblablement être assimilée à une relation purement causale (i.e., naturaliste).

Il me paraît difficile de trouver mieux que (23) ou (24), aussi longtemps qu'on s'en tient à l'idée que la relation de ressemblance doit intervenir dans les conditions de vérité des attributions d'états intentionnels. Mais je suis fort tenté d'abandonner cette contrainte puisque cela permettrait de caractériser les diverses interprétations non solipsistes de (9) de façon beaucoup plus naturelle:

(25) Fido a une inscription en mode $C$ qui est vraie ssi il y a un chat dans l'arbre devant lui (= Fido, ou M. Lambert)

(26) Il y a un arbre devant Fido (ou M. Lambert) et Fido a une inscription en mode $C$ qui est vraie ssi il y a un chat dans cet arbre.

Ces deux formulations sont manifestement assez proches de ce qu'on pourrait obtenir en appliquant l'analyse parataxique de Davidson, qui ne convient en effet qu'aux attributions d'états intentionnels considérés comme des identifications de leur objet :

(27) Fido a une inscription en mode $\mathrm{C}$ de ceci. Il y a un chat dans l'arbre devant lui.

(28) Il y a un arbre devant Fido (M. Lambert) et Fido a une inscription en mode $\mathrm{C}$ de ceci. Il y a un chat dans cet arbre. 
Je suis néanmoins enclin à préférer (25)-(26), parce qu'elles évitent les trois objections soulevées par Baldwin (1982:273) et d'autres à l'encontre de l'analyse parataxique. La première est que (27) et (28) impliquent l'existence d'une énonciation de la part du locuteur, ce qui n'est pas le cas de la phrase originale (9), ni d'ailleurs de (25) ou (26). La seconde est l'argument de Church, selon lequel une traduction littérale de (27) ou (28) ne produit pas une interprétation correcte de (9). On voit immédiatement que (25) et (26) échappent à cette objection.

La troisième objection consiste à souligner que l'inscription de Fido aurait pu avoir les mêmes conditions de vérité que l'énonciation de « il y a un chat dans l'arbre devant lui » mentionnée en (27), ou que l'énonciation de «il y a un chat dans cet arbre» mentionnée en (28), sans cependant avoir les conditions de vérité qu'elle a en fait. Cela vient de ce qu'une inscription ou une énonciation n'a telles ou telles conditions de vérité que de façon contingente. Mais sil'inscription de Fido n'avait pas les conditions de vérité qu'elle a en fait alors (9) serait fausse (c'est-à-dire, on ne pourrait dire de lui qu'il croit qu'il y a un chat dans l'arbre devant lui). Par conséquent, il est possible que (27) ou (28) soient vraies bien que (9) soit fausse. On voit immédiatement, encore une fois, que cette objection ne s'applique pas à (25) et (26) puisqu'elles spécifient directement les conditions de vérité de l'inscription de Fido sans mentionner aucun énoncé (ni par conséquent utiliser la relation «avoir les mêmes conditions de vérité que »).

Je conclurai cette discussion en remarquant que si une interprétation non solipsiste d'une attribution d'état intentionnel (singulier) correspond bien à ce qu'on appelle généralement une interprétation de re, il serait abusif d'assimiler une interprétation solipsiste à une interprétation de dicto (aussi longtemps du moins qu'on se limite aux états intentionnels privés), au sens où ce terme est généralement utilisé. Les remarques qui précèdent suggèrent au contraire qu'il n'y a vraisemblablement pas d'interprétation de dicto des attributions d'états intentionnels singuliers ${ }^{5}$.

Département de philosophie, Université du Québec à Trois-Rivières

5. Une communication basée sur une version abrégée de ce texte a été présentée à l'ACP en 1985. Je remercie vivement Storrs McCall pour ses commentaires. 


\section{BIBLIOGRAPHIE}

BALDWIN, Thomas (1982) : «Prior and Davidson on Indirect Speech», Phil. Studies 42, 25-28.

BURGE, Tyler (1979) : "Individualism and the Mental », Midwest Studies IV, 73-122.

(1982a) : «Other Bodies », Woodfield ed. (1982), 97-120.

(1982b) : «Two Thoughts Experiments Reviewed », Notre-Dame J. of Formal Logic, 23, 284-293.

CASTAÑNDA, Hector-Neri (1967) : «Indicators and Quasi-Indicators », American Phil. Quart. 4, 85-100.

DENNETT, Daniel C. (1978) : Brainstorms, Brighton, Harvester Press. (1982) »: «Beyond Belief », Woodfield ed. (1982), 1-96.

ENGEL, Pascal (1984a) : «Functionalism, Belief and Content», Torrance ed. (1984) 51-6.

(1984b) : “Croyance, dispositions et probalités», Rev. Phil. de la France et de l'étranger, 174, 401-426.

FIELD, Hartry (1978) : “Mental Representation», Erkenntnis 13, 9-61.

FODOR, Jerry (1975): The Language of Thought, New York, Crowell. (1980) : «Methodological Solipsism Considered as a Research Strategy in Cognitive Psychology », Fodor (1981), 225-253. (1981) : Representations, Brighton, Harvester Press.

KAPLAN, David (1977) : Demonstratives, manuscrit non publié.

LAURIER, Daniel (1987) : "L'individuation des états psychologiques et le statut de la psychologie populaire ", Recherches sur la philosophie et le langage, 8 , 49-73.

LEWIS, David (1979) : «Attitudes de dicto and de se", Lewis (1983), 133-159. (1983) : Philosophical Papers I, Oxford U. P.

OWENS, Joseph (1983) : «Functionalism and Propositional Attitudes », Noûs 17, 529-549.

PUTNAM, Hilary (1975): «The Meaning of "Meaning", Putnam (1975b), 215-271.

(1975b) : Mind, Language and Reality, Cambridge, Cambridge U. P. (1981) : Reason, Truth and History, Cambridge, Cambridge U. P.

SEARLE, John (1983) : Intentionality, Cambridge, Cambridge U. P.

TORRANCE, T. ed. (1984): The Mind and the Machine: Philosopbical Aspects of Artificial Intelligence, Chichester, Ellis Horwood.

WOODFIELD, Andrew ed. (1982): Thought and Object, Oxford, Clarendon Press.

STICH, Stephen (1978) : «Autonomous Psychology and the Belief-Desire Thesis ", The Monist 61, 573-591. 
(1978b) : "Beliefs and Subdoxastic States ", Phil. of Sc. 45, 499-518.

(1982): «On the Ascription of Content», Woodfield ed. (1982), $153-206$

(1983): From Folk Psycbology to Cognitive Science: The Case against Belief, Cambridge, Mass., MIT Press. 